\title{
The Impacts of Stress on Economic Decisions
}

\author{
Zachary P. Hohman ${ }^{1}$, Darren Hudson ${ }^{2,3 *}$, Ryan Williams ${ }^{2,3}$, Breanna N. Harris ${ }^{4}$, Jessica L. \\ Alquist $^{5}$, Donna Mitchell ${ }^{2}$, Elizabeth M. Niedbala ${ }^{5}$, Mindi Price ${ }^{5}$
}

\begin{abstract} that lowers overall gains but increases the probability of success.

\section{Keywords}

Stress - Economic decisions - Ultimatum games - Bidding - Cold pressor

${ }^{1}$ Social Identity Theory and Health Laboratory, Department of Psychological Sciences, Texas Tech University

${ }^{2}$ Department of Agricultural and Applied Economics, Texas Tech University

3 Texas AgriLife Research - Lubbock

${ }^{4}$ Department of Biological Sciences, Texas Tech University

${ }^{5}$ Department of Psychological Sciences, Texas Tech University

*Corresponding author: Email: darren.hudson@ttu.edu
\end{abstract}

The rational choice model presumes that individuals are rational and make optimizing decisions based on available information. Theory suggests that lack of information and risk (and risk perceptions) can alter decisions from the static perfect information case, but do not necessarily result in irrational decisions. Stress is another factor that may alter our perceptions and increase cognitive loading (increase the cost) of decision-making. Here, we use an experiment to induce stress and employ a simple ultimatum bargaining game to determine whether stress impacts economic decisions. Our results indicate that those exposed to stress (psychological, uncertainty or physiological, cold pressor task) significantly lower their gains (become less aggressive in bidding) than the control group. These results suggest that stress does, in fact, change behavior and leads to "hedging" behavior

\section{Introduction}

Stress in daily economic decisions can be quite pervasive: single parents balancing work and children; high-pressure sales tactics of car dealers; hungry families and terrorist recruiting; etc. The standard rational choice model presumes that individuals evaluate information systematically and the neoclassical economic model further assumes that individuals make choices that maximize their own benefit. That is, they rationally evaluate costs and benefits of actions. However, data do not always seemingly follow these predictions. These models work well for situations where people have access to full information or where outcomes are known, but when level of risk (Sandmo, 1971) or cognitive load increase (Tversky and Kahneman, 1974), results no longer conform to the model. And even emotional states such as sadness and dietary choices (Strang et al., 2017) can impact economic decisions (Harlé and Sanfrey, 2007). Thus, external factors and emotional states can influence how individuals evaluate cost and benefit trade-offs and can play an important role in understanding how individuals make economic decisions (Tversky and Khaneman, 1974) and, thus, have important implications for how policy is designed and implemented. One of the most ubiquitous factors experienced by humans is stress (Jackson, 2013). Generally, stress is defined as an external load on a physiological or psychological system/individual (Lazarus, 1993); however, the stimulus (internal or external), the perception (sensory and cognitive processing) and the physiological and behavioral responses to stressors are also important (Monroe, 2008). Stressors, inductors of stress, can be acute (short-term) or chronic (long-term), physiological (e.g., pain, illness, injury, hunger) or psychological (e.g., fear, anxiety, uncertainty), and activate various physiological stress response systems (e.g., the hypothalamic-pituitary-adrenal axis and the sympathetic "fight or flight" nervous system). Individuals can experience multiple different stressors at any given time, and impacts can be additive. Stressors, and the body's physiological response to them, can alter many aspects of behavior, cognition, affect, feeding, and overall health (Sapolsky et al., 2000; Juster et al., 2010). Thus, we would hypothesize that stress influences an individual's capacity to process information and make economic decisions. Additionally, decision complexity in itself may be a stressor (think buying a house or investing in your retirement fund). Alternatively, relatively simple decisions (think meal planning decisions) may be exacerbated by external stressors. In either case, the stress leads to increased decision costs (diverted attention, interrupted or costly information gathering, etc.). These higher cognitive costs likely lead to employing heuristics to reduce decision cost. Thus, it is reasonable to ask does exposure to stress change economic decision making? Or, more specifically, does stress change the way in which individuals evaluate costs and benefits?

Here, we aimed to determine if stress impacts economic decision making in a controlled setting where stress stimuli are measured and controlled. Economic decisions are easily measured, and all factors can be evaluated with known re- 
sults in the literature. Specifically, we were interested in how stress affected economic decision making in the ultimatum game (UG) - a commonly used experimental instrument in economics and psychology. In the UG, participants are asked to split an amount of money with an ostensible partner. Participants are told that the other participant can accept or reject their offer. If the offer is rejected, neither person receives any money. Previous research has found that when cognitive resources are low due to a previously demanding task, participants offer a number closer to an even split in the UG than when cognitive resources are not low (Halali, Bereby-Meyer, and Ockenfels, 2013). Here, we predict that when faced with stress, both psychological and physiological, individuals will change their response to a stimulus compared to the same situation without incidental (external) stress. Specifically, we predict that both forms of stress will make participants less risk-seeking and will promote a 50/50 split more frequently than in a control with no stress. This paper uses a combined psychological/economic experiment to test whether stress leads to alterations of behavior in simple economic decisions. The results will help researchers (and policy-makers) better understand how stress may be impacting decisions and account for those effects in planning research and implementing policy.

\section{Previous Literature}

\section{Basic Ultimatum Game}

The basic structure of the Ultimatum Game (UG) . ${ }^{1}$ The game formulation is such that the money-maximizing Nash equilibrium should be for both parties to accept any positive offer because it makes both parties better off. However, the bulk of the literature has found subjects typically split the gains evenly (or near evenly). This outcome led to a veritable cottage industry attempting to explain the apparent anomaly. Altruism, expectations, fairness, reciprocity, etc. were all examined (see, e.g., Suleiman, 1996; Eckel and Grossman, 1996; Falk and Fischbacher, 2006), as well as more complex issues like bounded rationality (Camerer and Fehr, 2006; Lusk and Hudson, 2004). Lusk and Hudson (2010) even inverted the UG to bargaining over losses and found behavior more consistent with the Nash equilibrium suggesting that behavior depends on whether respondents are sharing gains or losses. Some studies have controlled for outside risk-taking behavior

\footnotetext{
${ }^{1}$ This economic-based game is a standard lab procedure that can be used to assess risk taking (Takahashi, 2007). The proposer is allocated $\$ 10$ per round and is asked to make a proposed split of the $\$ 10$ with their responder (any amount from $\$ 0$ to $\$ 10$ ). If the responder accepts the offer, the split is made, and the participants are paid. If the responder rejects the offer, neither the proposer nor the responder is paid. Here, participants were placed at a computer and told that they would be playing a game against another student, and that the computer would randomly assign them to be the proposer or the responder in each round. In reality, participants were playing against the computer, were always assigned to be the proposer, and the computer was programmed to accept any offer over $\$ 0$. The participants played a total of 8 rounds. An eight-sided die was used to randomly choose one of the session rounds as the "binding" pay round. We summed the amount of money offered by the proposer across the eight rounds.
}

(Takahashi, 2007) but inducing stress within the game and controlling for baseline risk-taking behavior, as we do here, is beneficial for understanding how subjects make decisions following the introduction of stress. The studies above (and many others) using the UG have mixed outcomes in explaining divergences from the Nash equilibrium or the 50/50 split. A primary advantage of the UG is its simplicity, which, coupled with the plethora of findings, makes it simple to draw comparisons with the broader literature.

Stress is a ubiquitous and unavoidable part of life and results from exposure to stressors. Stressors can be acute (shortterm) or chronic (long-term), and range from psychological (a hostile boss, traffic, work deadlines, interpersonal relationships, financial troubles, uncertainty etc.) to physiological (thirst, hunger, injury, cold, etc.); but regardless of their nature, they elicit behavioral and physiological responses which aid individuals in coping with and appropriately responding to the situation (e.g., see Figure 1 in McEwen et al., 2015).

The most commonly measured biological responses to stress are the hypothalamic-pituitary-adrenal (HPA) axis and the sympathetic nervous system (e.g., the fight or flight response). The HPA axis and the sympathetic nervous system respond to a variety of stressors, whether physiological or psychological, and are present in all vertebrate animals, helping organisms respond to and cope with challenging conditions (Sapolsky et. al., 2000; Denver, 2009; Reeder and Kramer, 2005). Stress, and the hormones associated with the stress response (e.g., glucocorticoids, epinephrine), can alter many aspects of behavior and physiology, including cognitive processing and decision making in humans and animals (Sapolsky et al., 2000; Lupien et al., 2005; Greenberg et al., 2002).

The literature provides clear support for the biological linkage between stress and behavioral responses. For example, stressors - particularly predators - elevate glucocorticoids and can increase vigilance behavior, decrease feeding, and decrease risk-taking in prey animals; all behaviors which help animals avoid becoming a meal for a predator (Harris and Carr, 2016). This well-documented risk-reducing response to acute stressors has also been shown in humans, but results can differ based on participant sex (Mather and Lighthall, 2012) and age (Mather et al., 2009). Specifically, higher risk-taking, via the Balloon Analogue Risk Task (BART) ${ }^{2}$, was noted in men when completed 15 min after acute stress (3-min cold pressor), whereas lower risk-taking was noted in women; but, when data were combined and hormonal response was added, higher cortisol (the human glucocorticoid hormone) increases were correlated with less risky behavior (Lighthall et al., 2009), suggesting high cortisol is associated with decreased risk. This relationship between stress hormones and risk was also noted

\footnotetext{
${ }^{2}$ The BART is a validated measure of risk taking (Lejuez et al., 2002; Benjamin Robbins, 2007). In the BART, participants pump up a virtual balloon while receiving money for each pump. Participants can push a "Collect $\$ \$ \$$ " button at any point to collect the money from that balloon. However, the balloon may burst on any given pump, and any money not "collected" before the burst is lost.
} 
in a study using the Iowa Gambling Task ${ }^{3}$ : male and female subjects with higher baseline cortisol made more risk-aversive decisions and earned more money in the game when compared to individuals with lower baseline cortisol (van Honk et al., 2003). Thus, stress and stress hormones influence riskiness, causing people to become more risk averse. One form of stress we were interested in testing was the experience of uncertainty. Uncertainty is experienced when someone lacks information about a situation (Knight, 1921). Because uncertainty can make it difficult to operate in one's environment, it is often an aversive and stressful experience (Hirsh, Mar, Peterson, 2012; Hogg, 2014, though see Bar-Anan, Wilson, and Gilbert, 2009 and Wilson, Centerbar, Kermer, and Gilbert, 2005 for exceptions). Previous research has shown that uncertainty can also be cognitively demanding and can reduce success at subsequent effortful tasks (Alquist et al., 2018; Milkman, 2012). Because previous research has shown that people offer their ostensible partner in the UG a 50/50 split when they have been cognitively taxed (Halali, Bereby-Meyer, Ockenfels, 2013), we predict that participants exposed to uncertainty will offer more (closer to the 50/50 split) than participants in a control condition of no uncertainty. Uncertainty should decrease riskiness.

Risk-taking as discussed above is of importance in understanding behavior, but risk-taking behavior can be confounded between riskiness (objective probabilities of events occurring) and risk preferences (risk aversion). The potential for confounding complicates interpreting response. Here, we focus on a simple division of economic surplus with a well-known Nash equilibrium that is not dependent on risk preferences directly. Additionally, because there is a large body of literature using the UG with a fixed money-maximizing strategy, we can place our results in the larger literature without worry about context-specific or risk-preference specific results. But, we will control for risk preferences within subjects using a risk preference elicitation scale.

To summarize, the literature clearly indicates that stress, and specifically uncertainty, generates very specific, predictable biological responses. Those biological responses, in turn, are expressed in behavioral changes, particularly movements toward more risk-averse choices. Our hypothesis here is that those biological responses also manifest themselves in observable economic decisions as well; namely, exposure to stress/uncertainty will lead to larger offers in the UG (taking less of the surplus), thereby reducing the risk of rejection. In this sense, we are proposing that stress induces a larger trade-off of economic surplus for certainty in outcome.

\footnotetext{
${ }^{3}$ The Iowa Gambling Task asks participants to make advantageous choices by drawing from four different decks of cards with unknown advantages and disadvantages (Bechara et al., 1994; Bechara et al., 1997). Two of the decks are ultimately advantageous while two are ultimately disadvantageous, the faster that participants can determine which decks are advantageous determines how much money they make in the game.
}

\section{Methods}

\section{Experiment Setup/Recruitment}

Participants were recruited to participate in the study using an introduction to psychology course participant pool. Those who participated in the study received partial course credit. Participants were randomly assigned to one of the three experimental conditions (uncertainty, certainty, and cold pressor stress). To manipulate uncertainty (with certainty the control condition), participants were asked to write down three things that make them feel uncertain about themselves, their future, or their place in the world (this is a validated method for manipulating uncertainty/certainty; see Hohman and Hogg, 2015). For the cold pressor, participants placed their hand in ice water for 3 minutes (this is a known method of inducing stress, see Brady et al., 2006; Lighthall et al., 2009; Niedbala et al., 2018). After the stressor manipulation participants played the UG and after playing the game they answered questions measuring their risk level and demographics. At the end of the study, a random round of the UG was chosen as the pay round and participants were paid how much they won in that round. ${ }^{4}$

\section{Ultimatum Game: Setup, Rounds, Payoffs}

After the stress manipulation participants played 8 rounds of the UG . Participants were told that they would play a game with another person (electronically in another part of the university) and that there would be a proposer and a decider. The proposer would be the person who proposes how to split $\$ 10$ between the two, and the decider is the person who gets to decide to accept the offer. For this game participants always played as the proposer, though they were told that at the beginning of each round that the roles of proposer versus responder would be randomly assigned. Participants were always playing against a computer and the computer would accept all offers unless the proposer chose to keep all of the money for themselves (so an offer of 0 was rejected, all other offers were accepted); participants were not aware that all offers over 0 would be accepted. Participants were told that at the end of the study one of the eight rounds would be chosen as the pay round, and that the money they won in that round would be the money that they received. At the end of the study participants rolled an eight-sided die to determine the pay round.

\section{Risk Measure}

To control for individual differences in risk taking, at the end of the study participants completed a validated risk-taking measure (Weber, Blais, and Betz, 2002). This is a 40-item scale that asks participants about their risk-taking across multiple domains (e.g., financial, cheating, etc.) on a 5-point Likert scale ( 1 very unlikely to do behavior, 5 very likely to do behavior), e.g. items "Betting a day's income at a high

\footnotetext{
${ }^{4}$ Saliva samples were also collected from each participant before and after the experiment to measure any changes in cortisol and testosterone levels. Those data are being analyzed and reported elsewhere.
} 
stake poker game", "Illegally copying a piece of software", and "Passing off someone's work as your own". To create a single risk-taking measure we took the average of the 40 items, $\alpha=0.83$.

\section{Results}

\section{Sample Characteristics}

The total sample and treatment subgroup descriptive statistics are shown in Table 1. The average gain across all rounds and respondents of $\$ 5.72$ indicates that the average offer was slightly less than $\$ 5$, and the median gain of $\$ 5.63$ indicates a relatively normal distribution of offers as reflected in Figure 1 .

Overall, the data show that respondents were, on average, slightly more aggressive than the typical 50/50 split observed in other UG analyses, but certainly far from the predicted Nash equilibrium of extracting the full surplus (very small offers). Overall, the sample was predominantly college freshman, and largely (92\%) female. A bit over half of the sample viewed their earnings from the experiment as being derived through luck rather than skill (see Table 1).

Table 1. Descriptive Statistics for Key Variables in the Overall Sample and Each Treatment Sub-Group (Not Adjusted for Male/Female Proportions)

\begin{tabular}{|c|c|c|c|c|c|}
\hline Variable & Mean & Median & Standard Deviation & Minimum & Maximum \\
\hline & \multicolumn{5}{|c|}{ Overall Sample $(\mathrm{n}=152)$} \\
\hline Gain & 5.72 & 5.63 & 1.42 & 2.00 & 9.25 \\
\hline Age & 18.75 & 18.00 & 1.73 & 17.00 & 30.00 \\
\hline Male & 0.18 & N/A & 0.38 & 0.00 & 1.00 \\
\hline "Lucky" & 0.56 & $\mathrm{~N} / \mathrm{A}$ & 0.50 & 0.00 & 1.00 \\
\hline \multirow[t]{2}{*}{ Risk } & 3.25 & 3.19 & 0.65 & 2.00 & 5.45 \\
\hline & \multicolumn{5}{|c|}{ Certainty $($ Control) $(\mathrm{n}=49)$} \\
\hline Gain & 5.83 & 5.75 & 1.25 & 3.13 & 8.75 \\
\hline Age & 18.51 & 18.00 & 0.98 & 17.00 & 22.00 \\
\hline Male & 0.10 & $\mathrm{~N} / \mathrm{A}$ & 0.30 & 0.00 & 1.00 \\
\hline "Lucky" & 0.60 & N/A & 0.49 & 0.00 & 1.00 \\
\hline \multirow[t]{2}{*}{ Risk } & 3.15 & 3.13 & 0.55 & 2.00 & 4.33 \\
\hline & \multicolumn{5}{|c|}{ Uncertainty (Stress) $(n=54)$} \\
\hline Gain & 5.73 & 5.56 & 1.72 & 2.00 & 9.13 \\
\hline Age & 19.15 & 18.00 & 2.12 & 18.00 & 28.00 \\
\hline Male & 0.24 & N/A & 0.43 & 0.00 & 1.00 \\
\hline "Lucky" & 0.63 & $\mathrm{~N} / \mathrm{A}$ & 0.49 & 0.00 & 1.00 \\
\hline Risk & 3.29 & 3.21 & 0.64 & 2.03 & 4.93 \\
\hline
\end{tabular}

The average risk measure in the study was a 3.25 (on a 5 point scale, $\mathrm{SD}=.65$ ). This suggests that participants were in the middle of the scale or moderately risky. Risk did not differ by treatment. We entered risk as a covariate in the analyses to control for the risk preferences of the subject, and those preferences did have a significant impact on decisions by subjects.

The data in Table 1 (also reflected in Figure 2) show that both of the stress treatments resulted in lower median gains (higher median offers) when compared to the control. ${ }^{5}$

\footnotetext{
${ }^{5}$ These results are based on the entire sample of data across all rounds.
}

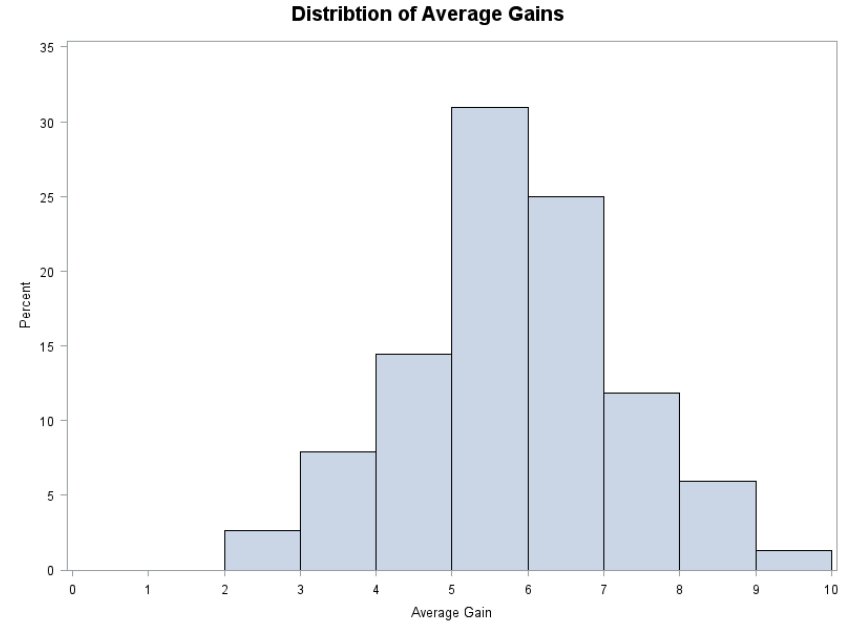

Figure 1. Distribution of Average Gains Across the Entire Sample

However, a simple ANOVA $(\mathrm{F}=0.29)$ and median scores test $\left(\chi^{2}=0.26\right)$ of the mean gains suggests no statistical difference between treatments. But, note that the proportion of males is underrepresented in the control by nearly half versus the sample mean and over-represented (by nearly half) in the uncertainty treatment relative to the sample mean. Thus, to control for the differences in representativeness, the average gains were divided by the ratio of each treatment's proportion to the sample mean $(0.57$ for the control, 1.37 for the uncertainty, and 1.06 for the cold pressor treatments, respectively). After controlling for the differences in respondent characteristics, the resulting ANOVA $(\mathrm{F}=204.96)$ and median scores test $\left(\chi^{2}=72.03\right)$ suggested that the mean/median gains were different across treatments, with the control exhibiting the highest numerical gain.

A General Linear Model (GLM) was used to estimate the impacts of treatment effects and other variables on the average gain. Here, we use the self-reported risk composite measure (Risk) to control for the degree of risk aversion for each respondent. The dependent variable is the average gain adjusted for differences in treatment male proportions. The results are shown in Table 2.

The treatment effects and risk all significantly contribute to the reduction of the Error Sum of Squares. The parameter estimates show the relative impact of each variable. Here, the estimated coefficient for Risk suggests that as the composite risk index rises (risk aversion declines), respondents became more aggressive with their bidding (lower offers/larger gains), which is consistent with theoretical expectations. After controlling for the impacts of risk aversion, the results show that both stress treatments exhibited significantly lower gains (higher offers) relative to the control group. Both a simple median test of the gains data and likelihood ratio test of these parameters shows that the offers were not different between the two stress treatments. The difference from the control is important because it supports the hypothesis that stress, in 
Table 2. General Linear Model Estimates of Treatment Effects on Average Gains Controlling for Self-Reported Risk Composite Measure

\begin{tabular}{|c|c|c|c|c|c|}
\hline Source & df & Type I SS & Mean Square & F-value & P-value \\
\hline Risk & 1 & 906.53 & 906.53 & 372.38 & $<0.001$ \\
\hline Cold Pressor & 1 & 10.54 & 10.54 & 4.33 & 0.04 \\
\hline Uncertainty & 1 & 147.80 & 147.80 & 60.71 & $<0.001$ \\
\hline Source & df & Type III SS & Mean Square & F-Value & P-value \\
\hline Risk & 1 & 12.42 & 12.42 & 5.10 & 0.03 \\
\hline Cold Pressor & 1 & 144.68 & 144.68 & 59.43 & $<0.001$ \\
\hline Uncertainty & 1 & 147.80 & 147.80 & 60.71 & $<0.001$ \\
\hline Parameter & Estimate & \multicolumn{2}{|c|}{ Standard Error } & $\mathrm{t}$-value & P-value \\
\hline Intercept & 8.12 & \multicolumn{2}{|c|}{0.99} & 8.21 & $<0.001$ \\
\hline Risk & 0.39 & \multicolumn{2}{|c|}{0.17} & 2.26 & 0.03 \\
\hline Cold Pressor & -4.05 & \multirow{2}{*}{\multicolumn{2}{|c|}{$\begin{array}{l}0.53 \\
0.63\end{array}$}} & -7.71 & $<0.001$ \\
\hline Uncertainty & -4.88 & & & -7.79 & $<0.001$ \\
\hline $\mathrm{R}^{2}$ & 0.75 & & & & \\
\hline F-value & 145.81 & & & & \\
\hline
\end{tabular}

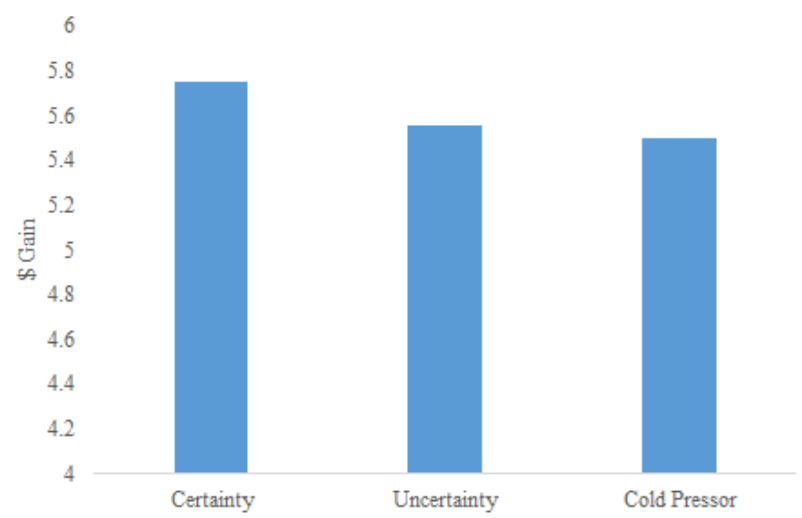

Figure 2. Median Gains By Treatment

general, does significantly alter economic decision-making with respect to uncertain opportunities for financial gains.

Here, respondents offered more money (took less gain) when under stress than compared with not. This result suggests, as expected, that stress increases opportunity costs of uncertain outcomes, thereby leading to choosing a more certain offer strategy. This should not be confused with risk aversion. The Risk variable shows that more risk seeking behavior leads to more aggressive offers, ceteris paribus. Rather, we suspect stress increases cognitive taxation and pushes respondents to a more heuristic "safe" response. That is, under stress, respondents appear more likely to take more certain gains rather than risk rejection, when the tolerance for risk is held constant. Interactions between risk and treatment effects were examined, but none were statistically relevant.

Finally, using the panel model, we controlled for the impacts of learning across rounds using a pooled OLS model - see Table 3 and Figure $3 .^{6}$

Table 3. Pooled Ordinary Least Squares Estimates of Respondent Gains Across Rounds

\begin{tabular}{|c|c|c|c|c|}
\hline Variable & Estimate & StandardError & t-value & p-value \\
\hline Intercept & 7.15 & 0.70 & 10.21 & $<0.001$ \\
\hline Risk & 0.38 & 0.12 & 3.17 & 0.00 \\
\hline Uncertainty & -4.86 & 0.43 & -11.30 & $<0.001$ \\
\hline Cold Pressor & -4.02 & 0.36 & -11.17 & $<0.001$ \\
\hline Round & 0.22 & 0.04 & 5.50 & $<0.001$ \\
\hline $\mathrm{R}^{2}$ & 0.44 & & & \\
\hline Root MSE & 3.02 & & & \\
\hline
\end{tabular}

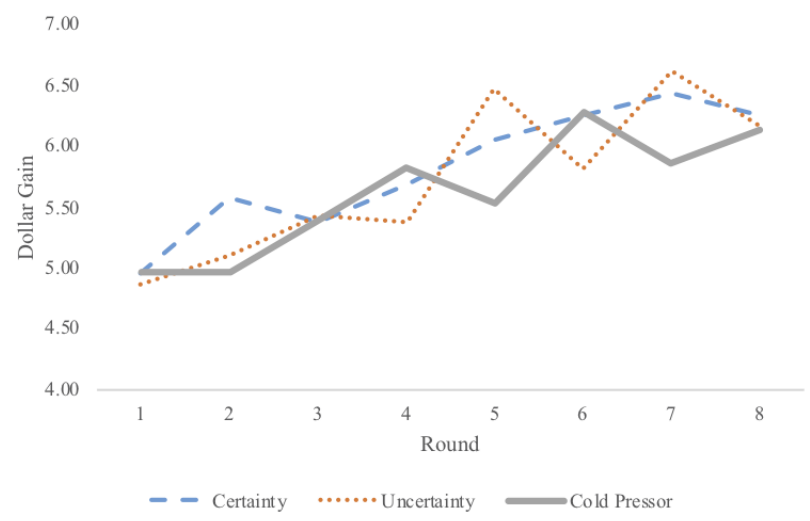

Figure 3. Average Gains By Round for Each Treatment

\section{Conclusions}

This research provides useful perspective on the role of stress in decision-making. Here, we see that stress, both psychological (uncertainty) and physiological (cold pressor), generally leads to forgoing higher portions of economic surplus to gain certainty even when holding risk tolerance constant. By disentangling riskiness from risk tolerance, we can conclude that it is the stress itself, and not the level of risk aversion, that is generating this decision. This result, then, helps disentangle arguments of "underperformance" in economic decisions from riskiness of choices versus risk aversion. More importantly, though, our results provide key evidence that stress does matter in economic decisions.

From a policy perspective, the impacts of stress should give pause to the standard assumptions that incentives and/or decisions made by agents in reaction to policy are free from external stressors. Can we expect, for example, that an unemployed person on food assistance will make optimal food choices as compared to others not facing the same stress? Our data suggest perhaps not. And if not, what implications might that have on how the policy is designed and implemented?

\footnotetext{
${ }^{6}$ One- and Two-way random effects models were also estimated, but results were not qualitatively different than presented here.
} 
Generally, our results suggest that policy-makers should carefully consider both the stress that policies induce as well as how individuals will likely react to policy while they are under the influence of other stressors.

While our results provide very interesting fodder for debate, it should be noted that our experiment is not dynamic. That is, while we can conclude that stress likely results in forgoing economic surplus, it does not say whether past decisions to give up surplus place a person in further stress which leads to further forgoing of surplus. That requires a whole different experimental framework, but is an interesting question for future research. Readers should also be cognizant of the fact that while the results are robust to a number of different model specifications and variables for analysis, the sample size is relatively small. Further, our operationalization of "stress" is quite specific, offering the advantages of validity and control, but forgoing some degree of realism to stresses experienced in the real world. Nevertheless, the pervasiveness of stresses in the real world and the impacts they are having on our results suggests we need a better, more complete understanding of how stress is likely to impact decision-making across a broader spectrum of stress types and types of economic decisions.

\section{References}

1. Alquist, J., R. Baumeister, I. McGregor, T. Core, I. Benjamin, and D. Tice. (2018). Personal conflict impairs performance on unrelated self-control task: Lingering costs of uncertainty and conflict. Journal of Experimental Social Psychology, 74:157-160.

2. Bar-Anan, Y., Wilson, T.D., Gilbert, T.D. (2009). The feeling of uncertainty intensifies affective reactions. Emotion, 9:123-127.

3. Bechara, A., Damasio, A., Damasio, H., and S. Anderson. (1994). Insensitivity to future consequences following damage to human prefrontal cortex. Cognition, 50:7-15.

4. Bechara, A., Damasio, H., Tranel, D., and A. Damasio. (1997). Deciding advantageously before knowing the advantages strategy. Science, 275:1293-1294.

5. Benjamin, A., and S. Robbins. (2007). The role of framing effects in performance on the Balloon Analogue Risk Task (BART). Personality and Individual Differences, 43:221-230.

6. Brady, K., A. Waldrop, A. Mcrae, S. Back, M. Saladin, H. Upadhyaya, R. Anton, and P. Randall. (2006). The impact of alcohol dependence and posttraumatic stress disorder on cold pressor task response. Journal of Studies in Alcohol, 67:700-706.

7. Camerer, C. and E. Fehr. (2006). When does "economic man" dominate social behavior. Science, 311(5757): 47-52.
8. Denver, R. J. (2009). Structural and functional evolution of vertebrate neuroendocrine stress systems. $A n$ nals of the New York Academy of Sciences, 1163(1):116.

9. Eckel, C. and P. Grossman. (1996). Altruism in anonymous dictator games. Games and Economic Behavior, 16(2):181-191.

10. Engert, V., Vogel, S., Efanov, S. I., Duchesne, A., Corbo, V., Ali, N., Pruessner, J. C. (2011). Investigation into the cross-correlation of salivary cortisol and alpha-amylase responses to psychological stress. Psychoneuroendocrinology, 36(9):1294-1302.

11. Falk, A. and U. Fischbacher. (2006). A theory of reciprocity. Games and Economic Behavior, 54(2):293315.

12. Greenberg, N., Carr, J. A., and Summers, C. H. (2002). Causes and consequences of stress. Integrative and Comparative Biology, 42(3):508-516.

13. Halali, E., Y. Bereby-Meyer, and A. Ockenfels (2013). Is it all about the self? The effect of self-control depletion on ultimatum game proposers. Frontiers in Human Neuroscience, 7:1-8.

14. Harlé, K. and A. Sanfrey. (2007). Incidental sadness biases social economic decisions in the Ultimatum Game. Emotion, 7:876-881.

15. Harris, B. N., and Carr, J. A. (2016). The role of the hypothalamus-pituitary-adrenal/interrenal axis in mediating predator-avoidance trade-offs. General and comparative endocrinology, 230:110-142.

16. Hellhammer, D. H., Wüst, S., and Kudielka, B. M. (2009). Salivary cortisol as a biomarker in stress research. Psychoneuroendocrinology, 34(2):163-171.

17. Hirsh, J.B., Mar, R.A., Peterson, J.B. (2012). Psychological entropy: A framework for understanding uncertainty-related anxiety. Psychological Review, 119:304320.

18. Hohman, Z. and M. Hogg. (2015). Fearing the uncertain: Self-uncertainty plays a role in mortality salience. Journal of Experimental Social Psychology, 57:31-42.

19. Hogg, M.A. (2014). From uncertainty to extremism: Social categorization and identity processes. Current Directions in Psychological Science, 23:338-342.

20. Jackson, M. (2013). The Age of Stress: Science and the Search for Stability. Oxford: Oxford University Press.

21. Juster, R. P., McEwen, B. S., and Lupien, S. J. (2010). Allostatic load biomarkers of chronic stress and impact on health and cognition. Neuroscience Biobehavioral Reviews, 35(1):2-16. 
22. Knight, F. H. (1921). Risk, Uncertainty, and Profit. Boston: Houghton Mifflin.

23. Lejuez, C., Aklin, W., Zvolensky, M., and C. Pedulla. (2003). Evaluation of the Balloon Analogue Risk Task (BART) as a predictor of adolescent real-world risktaking behaviours. Journal of Adolescence, 26:475479.

24. McEwen, B. S., Bowles, N. P., Gray, J. D., Hill, M. N., Hunter, R. G., Karatsoreos, I. N., and Nasca, C. (2015). Mechanisms of stress in the brain. Nature Neuroscience, 18(10):1353.

25. Monroe, S. M. (2008). Modern approaches to conceptualizing and measuring human life stress. Annual Reviews Clinical Psychology, 4:33-52.

26. Lazarus, R. (1993). From Psychological Stress to the Emotions: A History of Changing Outlooks. Annual Review of Psychology, 44(1993): 1-21.

27. Lighthall, N. R., Mather, M., and Gorlick, M. A. (2009). Acute stress increases sex differences in risk seeking in the balloon analogue risk task. PLoS One, 4(7), e6002.

28. Lupien, S. J., Fiocco, A., Wan, N., Maheu, F., Lord, C., Schramek, T., and Tu, M. T. (2005). Stress hormones and human memory function across the lifespan. Psychoneuroendocrinology, 30(3):225-242.

29. Lusk, J. and D. Hudson. (2004). Effect of monitorsubject cheap talk in ultimatum game offers. Journal of Economic Behavior and Organization, 54(3):439-443.

30. Mather, M., and Lighthall, N. R. (2012). Risk and reward are processed differently in decisions made under stress. Current Directions in Psychological science, 21(1), 36-41.

31. Mather, M., Gorlick, M. A., and Lighthall, N. R. (2009). To brake or accelerate when the light turns yellow? Stress reduces older adults' risk taking in a driving game. Psychological Science, 20(2):174-176.

32. Milkman, K. (2012). Unsure of what the future will bring? You may overindulge: Uncertainty increases the appeal of wants over should. Organizational Behavior and Human Decision Processes, 119:163-176.

33. Niedbala, E. M., Hohman, Z. P., Harris, B. N., Abide, A. C. (2018). Taking one for the team: Physiological trajectories of painful intergroup retaliation. Physiology and Behavior. 194:277-284.

34. Reeder, D. M., and Kramer, K. M. (2005). Stress in freeranging mammals: integrating physiology, ecology, and natural history. Journal of Mammalogy, 86(2):225-235.
35. Sandmo, A. (1971). On the theory of the competitive firm under price uncertainty. American Economic Review, 61(1):65-73.

36. Sapolsky, R. M., Romero, L. M., and Munck, A. U. (2000). How do glucocorticoids influence stress responses? Integrating permissive, suppressive, stimulatory, and preparative actions. Endocrine Reviews, 21(1):55-89

37. Strang, S., Hoeber, C., Uhl, O., Koletzko, B., Münte, T. F., Lehnert, H., ... Park, S. Q. (2017). Impact of nutrition on social decision making. Proceedings of the National Academy of Sciences, 114(25):6510-6514.

38. Suleiman, R. (1996). Expectations and fairness in a modified ultimatum game. Journal of Economic Psychology, 17:531-554.

39. Takahashi, T. (2007). Economic decision-making in ultimatum games by smokers. Neurological Endocrinology Letters, 28:659-661.

40. Tversky, A. and D. Kahneman. (1974). Judgement under uncertainty: heuristics and biases. Science, 185(4157):11241131.

41. van Honk, J., Schutter, D. J., Hermans, E. J., Putman, P. (2003). Low cortisol levels and the balance between punishment sensitivity and reward dependency. Neuroreport, 14(15): 1993-1996.

42. Weber, E. A. Blais, and N. Betz. (2002). A domainspecific risk-attitude scale: Measuring risk perceptions and risk behaviors. Journal of Behavioral Decision Making, 15:263-290.

43. Wilson, T.D., Centerbar, D.B., Kermer, D.A., Gilbert, D.T. (2005). The pleasures of uncertainty: Prolonging positive moods in ways people do not anticipate. Journal of Personality and Social Psychology, 88:5-21. 